\title{
Dissociative Electron Attachment to 2,3,6,7,10,11-Hexabromotriphenylene
}

$\underline{\text { Alexey A. Goryunkov }^{\mathrm{a} 1}}$, Nail L. Asfandiarov ${ }^{\mathrm{b}}$, Mars V. Muftakhov ${ }^{\mathrm{b}}$, Ilya N. Ioffe ${ }^{\mathrm{a}}$, Vera A.

Solovyeva ${ }^{a}$, Natalia S. Lukonina, ${ }^{\text {a Vitaliy Yu. Markov }}{ }^{\mathrm{a}}$, Rustam G. Rakhmeyev ${ }^{\mathrm{b}}$, Stanislav A.

Pshenichnyuk ${ }^{\mathrm{b}}$

${ }^{a}$ Department of Chemistry, Lomonosov Moscow State University, Leninskie Gory, 1-3, 119991,

Moscow, Russia

${ }^{\mathrm{b}}$ Institute of Molecule and Crystal Physics - Subdivision of the Ufa Federal Research Centre of the Russian Academy of Sciences, Prospekt Octyabrya, 151, 450075 Ufa, Russia

\section{Supporting Information}

\section{Table of contents}

Figure $S 1$. IR spectrum of hexabromotriphenylene $\quad S 2$

Figure $S 2$. HPLC-MS(APPI) data of hexabromotriphenylene sample $\quad S 3$

Figure $S 3$. HPLC-UV/Vis-MS(APPI) chromatograms, APPI mass spectra $\quad S 4$

and UV/Vis spectra of triphenylene.

Figure $S 4$. Anion and neutral yields as well as mean lifetime as a function $\quad S 5$

of incident electron energy in hexabromotriphenylene.

1 correspondence to Dr. Sc. A.A. Goryunkov, tel: +7 4959395373, fax: +7 4959391240, e-mail: aag@thermo.chem.msu.ru 


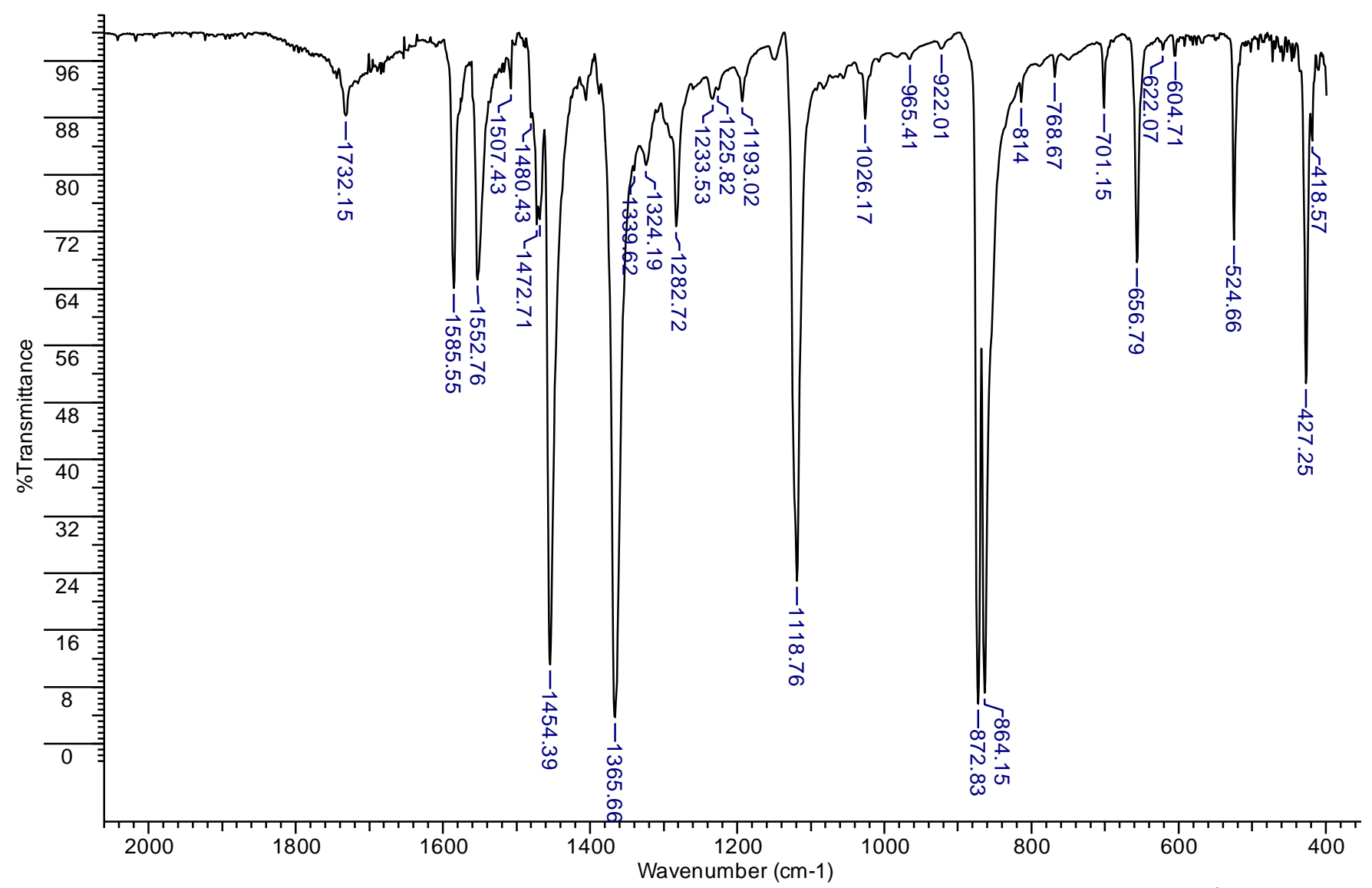

Figure $S 1$. IR spectrum of hexabromotriphenylene ( $\mathrm{KBr}$ tablet, resolution of $\left.2 \mathrm{~cm}^{-1}\right)$. 

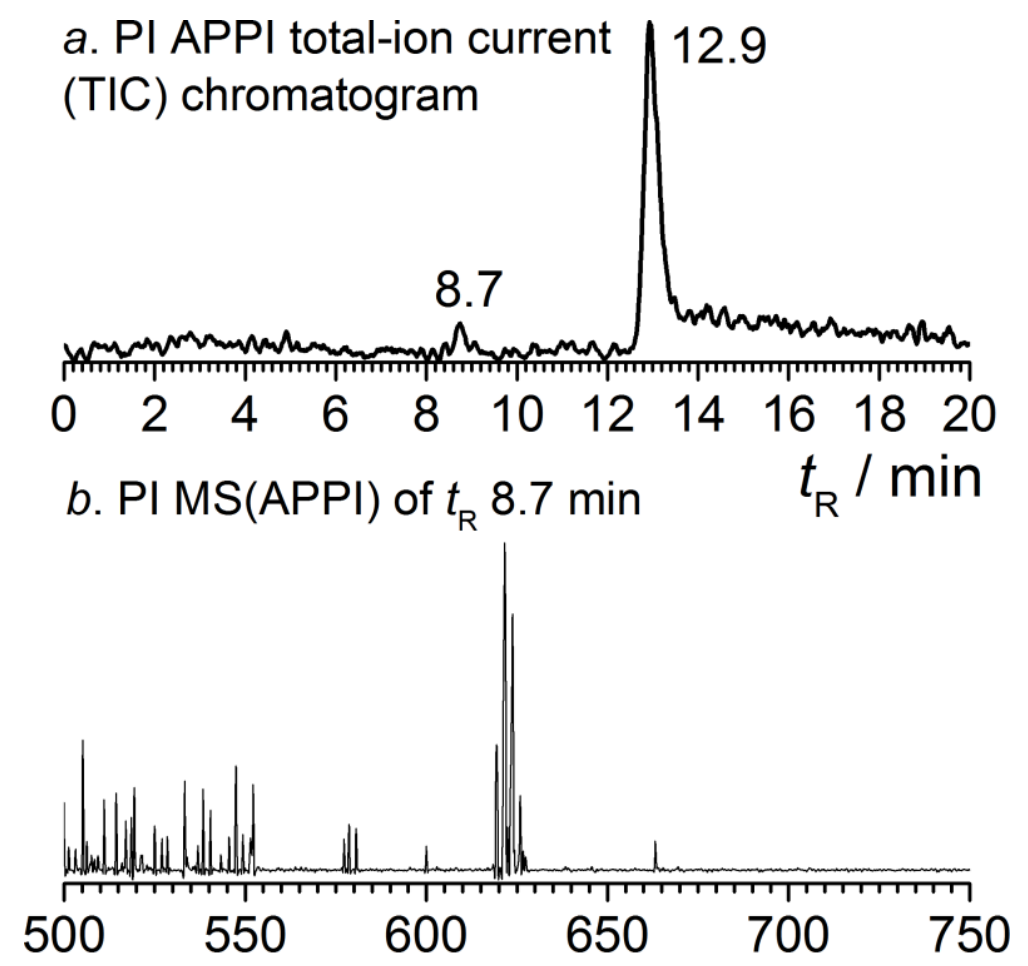

c. PI MS(APPI) of $t_{\mathrm{R}} 12.9 \mathrm{~min}$

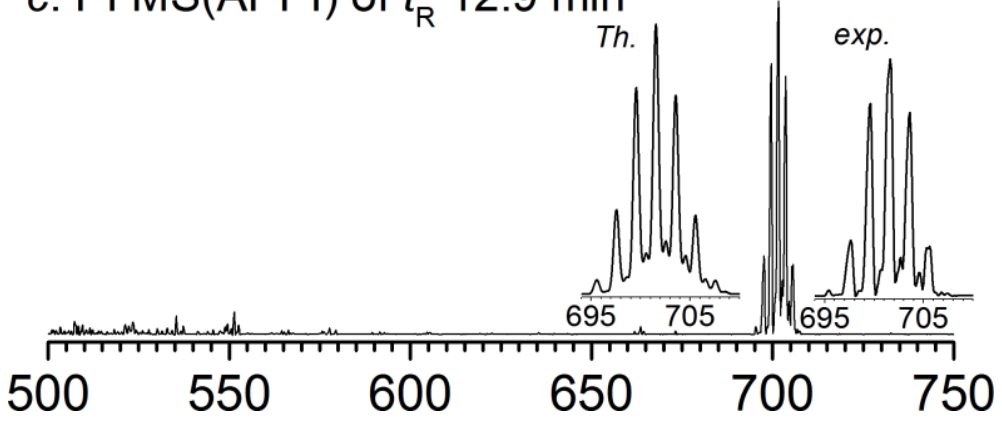

Figure S2. HPLC-MS data of hexabromotriphenylene sample: (a) Positive ion (PI) atmospheric-pressure photoionization (APPI) total-ion current (TIC) chromatogram; (b, c) PI APPI mass specta of minor and major fractions eluted at 8.7 and $12.9 \mathrm{~min}$, respectively (Cosmosil Buckyprep, $4.6 \mathrm{~mm} \times 25 \mathrm{~cm}$, hexane, $1 \mathrm{ml}$ $\left.\min ^{-1}, 23^{\circ} \mathrm{C}\right)$. 


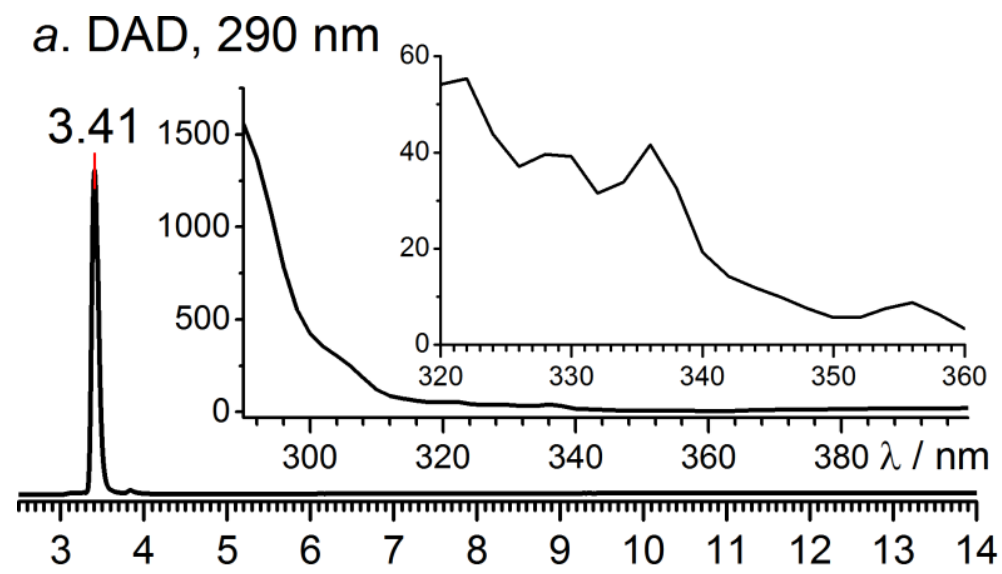

b. TIC

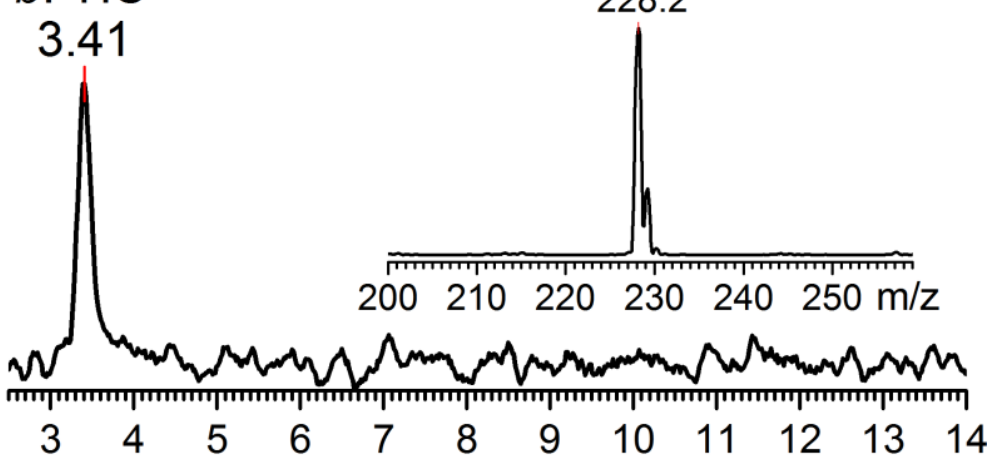

c. $\operatorname{EIC}(m / z=228.0)$

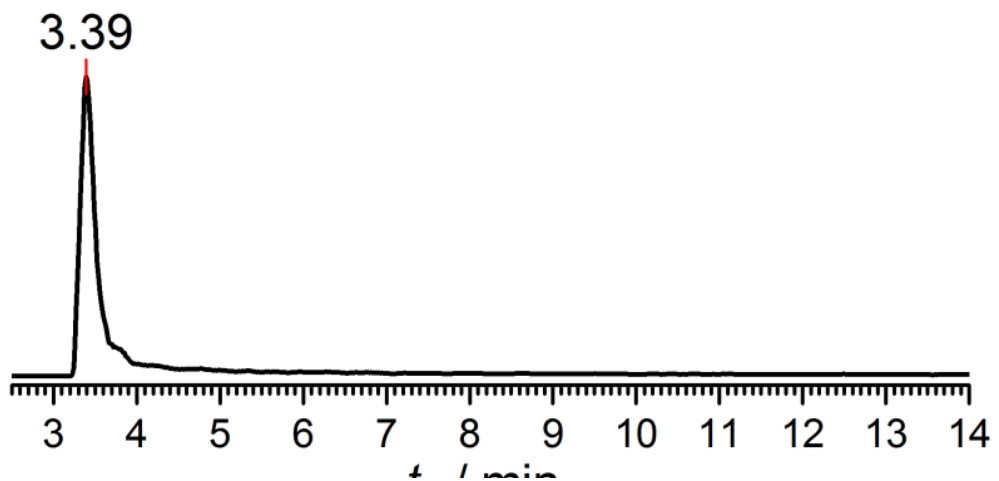

Figure S3. Simultaneously registered chromatogram of triphenylene (Cosmosil Buckyprep, $4.6 \mathrm{~mm} \times 25 \mathrm{~cm}$, toluene, $1 \mathrm{ml} \mathrm{min}^{-1}, 23{ }^{\circ} \mathrm{C}$ ): (a) HPLC-UV/Vis at $290 \mathrm{~nm}$ (UV/Vis spectrum is shown in inset), (b) HPLC-MS positive ion APPI total-ion current (TIC, APPI mass spectrum is shown in inset) and (c) extracted-ion current (EIC) chromatogram for $\mathrm{C}_{18} \mathrm{H}_{12}{ }^{+}$ion. 


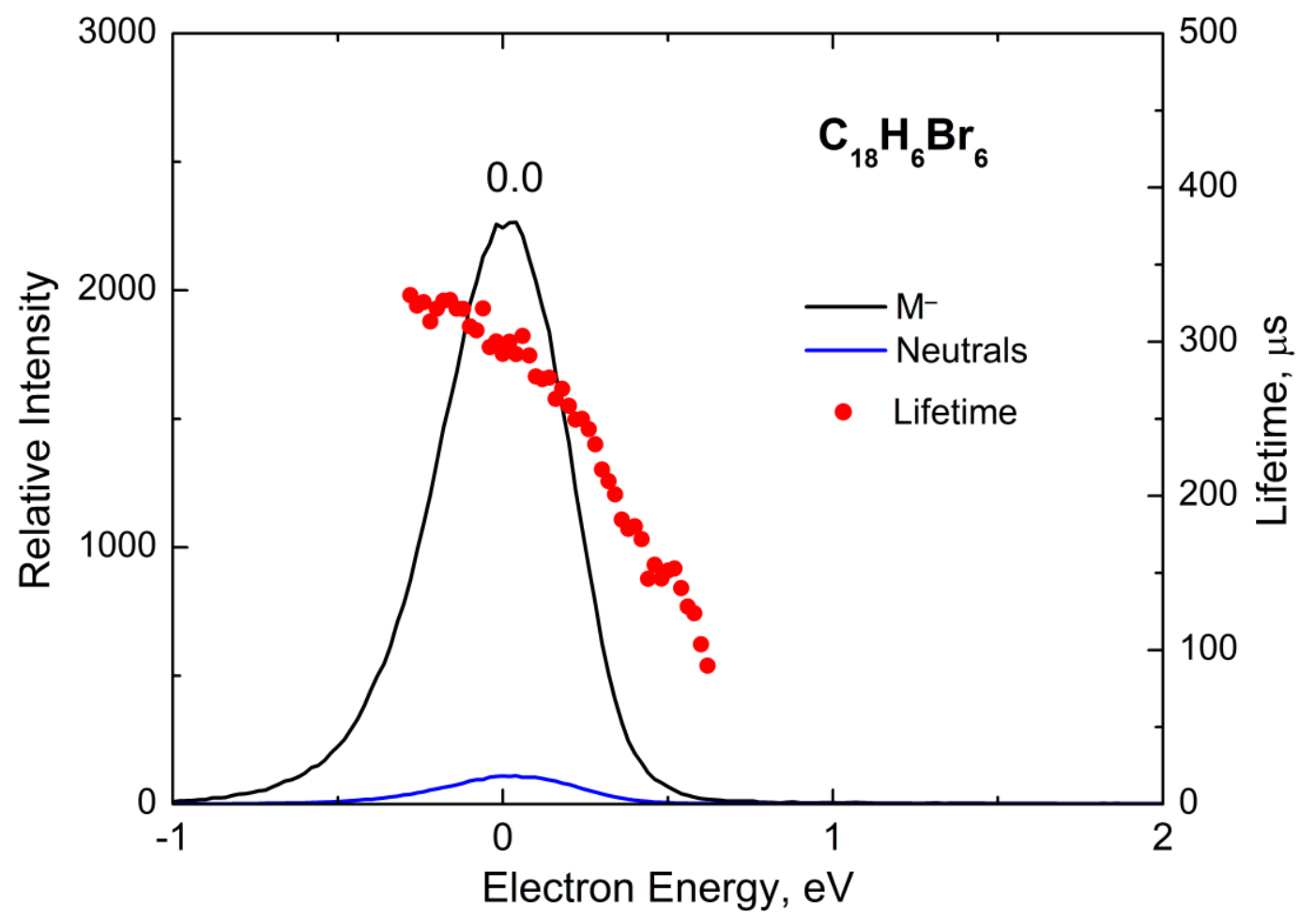

Figure S4. Anion (black line) and neutral (blue line) yield and mean lifetime (red circles) as a function of incident electron energy in hexabromotriphenylene. 